\title{
Predictive Controller Design for Networked Control System
}

\author{
Richa Sharma, Deepak Nagaria
}

\begin{abstract}
A networked control system where sensor ( ), controller () and an actuator () are interacting to the process model or system through a communication channel. The model reliability depends on time delay of data transmission from to , to , controller processing time and packet dropouts. In this proposed work, model based network system is designed to achieve the desired response of the controlled system by minimizing the network time delay and improves con-troller performance in term of stability. Time delay mainly occurs due to time sharing of the medium as well extra overload of signal coding and processing phase. The time delays could be steady, limited, or even random, depends upon protocol used in the network and hardware. The network predictive control tech-nique effectively works on random delay. The predictive controller is used to analyses the future output conditions of the system based on the previous values. The simulated result shows that if time delay increases, the response of the system degraded and therefore for improving it the correct choice of prediction and control horizon is needed. The designed networked control system based on predictive functional control is simulated using True Time tool on Matlab. The ob-tained outcomes from the proposed technique accomplishes that the technique is effective and feasible.
\end{abstract}

Keywords: Networked control system, predictive controller, communication delay, True-Time-MATLAB, Simulink, Stability, Reliability.

\section{INTRODUCTION}

A networked control system (NCS) is a closed loop system inside which data are transmitted over the correspondence system or channel. By introducing the communication network in a closed loop, analysis of the system becomes more complex. The main parts of the networked control system are sensor, controller, and actuator and communication network. The sensor nodes have a responsibility to measure one or multiple outputs of plant and transmitted this measured value to communication network. The actuator nodes have the assignment of feeding measured values got over the network to plant. The controller node utilize plant outputs that it get from the sensor node to compute control outputs by a control calculation and send them to the actuator node to be connected to the plant. The information are travel over the communication network in form of packets. The complex implementation and delays are the main drawbacks of networked system. While NCSs have a number of engaging favorable advantages, for example, ease, decreased weight and control necessities, and straightforward

Revised Manuscript Received on November 08, 2019.

* Correspondence Author

Richa Sharma, ${ }^{1}$ Research Scholar, Department of Electronics Engineering, AKTU, Lucknow, U.P., India

Dr. Deepak Nagaria, Associate Professor, Department of Electrical Engineering, B.I.E.T, AKTU, Lucknow, U.P., India establishment and upkeep together with productive applications in industry, for example, activity and correspondence frameworks, aeronautics, and space flight the addition of the correspondence arrange in the closed loop make the investigation and design complicated [2]. The addition of network systems in control closed loop prompts to some unavoidable marvels, including irregular postponement and packets dropout, which could break down framework execution and may even make the system unstable. One of the real difficulties in NCSs is network delays that happens while exchanging information among system components associated with the communication system. Without considering these delays, the framework execution outlined can be corrupted, and the framework can even be shaky. There are two types of networked induced delays random and time varying. Network induced delay degrades the overall system performance.

To tackle this time delay issues, numerous strategies have been received, for example, the increased deterministic discrete-time model technique and ideal dropout compensator strategy. Many researchers have proposed a networked predictive control technique where they have considered the stability of NCS. In [1] network predictive control method was used to minimize delay. An NDC (Network delay compensator) was constructed to compensate the network delays. Markovian jump system was used to formulate the system. This design was suitable for the distributed delay in the discrete domain. In [2] analyzed and synthesized the networked systems in presence of time delays and based on this analysis a predictive controller based design was proposed. This proposed predictive controller is useful to get desired performance and also guarantee the stability of the system. In [3] a lifting strategy was brought into the organized control system and another discrete-time switched model was recommended with the stress of postponement and packet dropout in the midst of the transmission. In [4] a model-based predictive NCSs (MBPNCSs) was proposed to avoid retransmissions due to data loss in the communication channel. This paper represented that by predicting the future states of plant data loss and delay of the network can be compensated. If time varying delay exceed the sampling time then the performance of the system degraded. So a fuzzy neural network to predict the sampling period of NCS was proposed in [5]. The fuzzy neural model was applicable to predict the communication time delay. In [6] introduced an intermittent feedback for the model based networked control system (MBNCS). They give state reaction and steadiness state of the system. In [7] an express model of the controlled plant was utilized by control engineering keeping in mind the end goal to limit the system stack while endeavoring to stay away from extreme response 
degradation. In [8] a novel predictive model was proposed for positive frameworks. The controller gain was calculated by linear programming. In this paper, gain was separated into two parts a non-positive part and a non-negative part. In [9] design a distributed model predictive controller for the asynchronous networked system. This type of controller uses asynchronous information to enhance the closed loop system in the networked control system.

In this paper, a predictive controller for the networked system is designing for improving the performance of the plant under the occurrence of time delay. The designing and analysis of predictive control system represent that the networked control system is dissimilar from conventional closed loop system. In proposed design CAN is used for data transmission from the predictive controller to plant and plant to the predictive controller.

This paper is organized such as follow section 2 portrays the general model of the networked system. Section 3 describes the overview of model predictive controller design, section 4 gives the designing of the model predictive controller. In section 5 simulation of the networked controlled servo control system in Matlab Truetime tool is given. At last, a few conclusions are made.

\section{NETWORK TIME DELAY MODEL}

The standard representation of networked control system is represented in Figure 2.This system consists of five major parts, a communication channel, plant, actuator, sensor , and controller. It is real time data transformation system. The delays occurrence when information transmitted from $\mathrm{C}$ to $\mathrm{A}$ and $\mathrm{S}$ to $\mathrm{C}$. This delays can be fixed or time varying, depending on the sampling period. NCSs have usually used two drive methods for control parts, time triggered and event triggered. In the time driven method the control components are triggered at fixed time stamp but in event triggered control components are triggered in presence of any event. Mostly sensor is time driven whereas actuator and controller is event triggered.

In Figure $2 \mathrm{C}$ to $\mathrm{A}$ and $\mathrm{S}$ to $\mathrm{C}$ time delays are lumped together. Here $\mathrm{u}(\mathrm{t})$ is the yield of controller and $\mathrm{y}(\mathrm{t})$ represent plant yield. The total time delay of the communication network is $\tau_{c a}+\tau_{g c}$. Where $\tau_{c a}$ is the delay from $\mathrm{C}$ to $\mathrm{A}$ and $\tau_{g c}$ is the delay from $\mathrm{S}$ to $\mathrm{C}$. This time delay degraded the system response. So to get desired response of the system networked predictive controller is presented.

\section{OVERVIEW OF MODEL PREDICTIVE CONTROLLER}

Model predictive controller (MPC) [10-11] enhances performance and stability of the networked system by holding the plant model inside the controller and ascertaining present and predicted control output to the plant for a few time ventures into the future at each sampling periods. On the off chance that a sensibly exact dynamic model of the process is accessible, show and current estimations can be utilized to anticipate future estimations of outputs. At that point, the proper changes in the input variables can be figured in view of both predictions and estimations. Model predictive control offers a few essential points of interest (1) the procedure demonstrate catches the static and dynamic collaboration between inputs, outputs, and unsettling influence factors, (2)

calculation of control signal with respect to ideal set points, and (3) exact model predictions can give prior notice of possible issues.

Figure 3 represents the structure of the model predictive controller. A process block utilizes to predict the present value of the outputs. Residuals are the difference between predicted outputs and process outputs. The anticipated outputs are used as a bit of two sorts of MPC estimations that are performed at each testing minute: set-point checks and control signal counts. Disproportion objectives on the data and output factors, for example, upper and lower limits, can be merged into either kind of calculation. In MPC, predictions depend on current estimations and desires without limits of the outputs [12].

In Figure 4. y, $\hat{y}$, $\mathrm{u}$ is real output, predicted output and manipulated input respectively for the system. At k sampling instant MPC technique figures an arrangement of different values of manipulated variables $u(k+i-1)$, (here $i=1,2$, 3...M). The information is confined steady after the $M$ control moves. The sources of information are figured with the goal that a plan of $\mathrm{P}$ predicted output $\mathrm{y}(\mathrm{k}-\mathrm{i})$, (here $\mathrm{i}=1$, 2. . . P) accomplish set point in a perfect way. The control estimations rely upon rebuilding an objective work.

\section{NETWORKED PREDICTIVE CONTROLLER DESIGN}

In light of the area of systems in a framework, there is a wide range of structures for NCSs. In a useful NCS, there exists time delay and information packets loss. The networked predictive controller considered to predict the states of the system [13].This type of controller generates the set of control predictions to achieve desired control response.

State space model for plant in discrete time

$x(k+1)=A_{n} x(k)+B_{n} u(k)$

$y(k)=C_{\mathrm{n}} x(k)$

In equation (1) and (2) x (k), $\mathrm{u}(\mathrm{k})$ and $\mathrm{y}(\mathrm{k})$ represent states, controlled system inputs and controlled outputs and A, B, C system matrix. According to receding horizon control, present plant information is useful for control and prediction.

By taking the distinction on two sides of equation (1) $x(k+1)-x(k)=A_{n}[x(k)-x(k-1)]+B_{n}[u(k)-$ $u(k-1)$

$\Delta x(k+1)=A_{n} \Delta x(k)+B_{n} \Delta u(k)$

In next step the incremented states $\Delta x(k)$ is connected to $\mathrm{y}(\mathrm{k})$.

(4)

$$
x(k)=\left[\Delta x(k)^{T} y(k)\right]^{T}
$$

$\mathrm{T}=$ Transpose of the matrix.

Output state

$$
y(k-1)-y(k)=C_{n}(x(k+1)-x(k))
$$

$C_{n} \Delta \mathrm{x}(\mathrm{k}+1)=C_{n} A_{n} \Delta \mathrm{x}(\mathrm{k})+C_{n} B_{n} \Delta \mathrm{u}(\mathrm{k})$

In equation (5) A, B, C is augmented model, which is useful for predictive controller design.

Expecting that at the sampling moment ki, ki $>0$, the state vector $\mathrm{x}(\mathrm{ki})$ gives the present plant information. The future control inputs are spoken to as 
$\Delta \mathrm{u}\left(k_{\mathrm{i}}\right), \Delta \mathrm{u}\left(k_{\mathrm{i}}+1\right), \ldots \ldots \ldots \Delta \mathrm{u}\left(k_{\mathrm{i}}+N_{c}-1\right)$

(6)

In equation (6) $N_{c}$ is the control horizon dealing with the number of parameters which are utilized to catch future control bearing. $N_{p}$ is the prediction horizon predicted future state for $N_{p}$ samples, for given data $x\left(k_{i}\right)$.

The predicted state variables are

$x\left(k_{i}+1 \mid k_{i}\right), x\left(k_{i}+2 \mid k_{i}\right), \ldots \ldots \ldots \ldots \ldots \ldots \ldots x x\left(k_{i}+\right.$ $\left.N_{p} \mid k_{i}\right)$

For model predictive controller control horizon must be not exactly or equivalent to prediction horizon. In the setting of state-space depiction, the future state factors are settled legitimately using the course of action of future control parameters [14]

$$
\begin{aligned}
& x\left(k_{i}+1 \mid k_{i}\right)=A_{n} x\left(k_{i}\right)+B_{n} \Delta u\left(k_{i}\right) \\
& x\left(k_{\mathrm{i}}+2 \mid k_{\mathrm{i}}\right)=A_{n} x\left(k_{\mathrm{i}}+1\right)+B_{n} \Delta u\left(k_{\mathrm{i}}+1\right) \\
& = \\
& A_{n}\left(A_{n} x\left(k_{i}\right)+B_{n} \Delta u\left(k_{i}\right)\right)+B_{n} \Delta u\left(k_{i}+1\right) \\
& =A_{n}^{2} x\left(k_{i}\right)+A_{n} B_{n} \Delta u\left(k_{i}\right)+B_{n} \Delta u\left(k_{i}+1\right) \\
& x\left(k_{i}+N_{p} \mid k_{i}\right)= \\
& A_{n}^{N_{D}} x\left(k_{i}\right)+A_{n}^{N_{D}-1} B_{n} \Delta u\left(k_{i}\right)+A_{n}^{N_{D}-2} B_{n} \Delta u\left(k_{i}+\right. \\
& \text { 1) } \ldots \ldots \ldots+A_{n}^{N_{D}-N_{c_{1}}} B_{n} \Delta u\left(k_{i}+N_{c}-1\right)
\end{aligned}
$$

The predicted output factors based on predicted state factors are

$$
y\left(k_{i}+1 \mid k_{i}\right)=C_{n} A_{n} x\left(k_{i}\right)+C_{n} B_{n} \Delta u\left(k_{i}\right)
$$$$
\begin{aligned}
& y\left(k_{\mathrm{i}}+2 \mid k_{\mathrm{i}}\right)=C_{\mathrm{n}} A_{n}^{2} \mathrm{x}\left(k_{\mathrm{i}}\right)+C_{\mathrm{n}} A_{\mathrm{n}} B_{\mathrm{n}} \Delta \mathrm{u}\left(k_{\mathrm{i}}\right)+ \\
& C_{\mathrm{n}} B_{\mathrm{n}} \Delta \mathrm{u}\left(k_{\mathrm{i}}+1\right)
\end{aligned}
$$

$$
\begin{aligned}
& y\left(k_{i}+N_{p} \mid k_{i}\right)=C_{n} A_{n}^{N_{D}} x\left(k_{i}\right)+C_{n} A_{n}^{N_{p}-1} B_{n} \Delta u\left(k_{i}\right)+ \\
& \ldots \ldots \ldots \ldots \ldots+C_{n} A_{n}^{N_{p}-\mathbb{N}_{c}} B_{n} \Delta u\left(k_{i}+N_{c}-1\right)
\end{aligned}
$$

(8)

The predicted output for single input and single output system (SISO)

$$
\begin{aligned}
& Y=F x\left(k_{\mathrm{i}}\right)+\Phi \Delta \mathrm{u} \\
& \text { Where } \quad \mathrm{F}=\left[\begin{array}{c}
\mathrm{C} A \\
\mathrm{C} \mathrm{A}^{2} \\
{ }^{-} \\
\mathrm{C} \mathrm{A}^{\mathrm{Np}}
\end{array}\right] \\
& {\left[\begin{array}{ccccc}
\mathrm{CB} & 0 & 0 & . & 0 \\
\mathrm{CAB} & \mathrm{CB} & 0 & : & 0 \\
\mathrm{CA} A^{2} \mathrm{~B} & \mathrm{CAB} & \mathrm{CB} & 0 & 0 \\
\vdots & \vdots & : & : & \vdots \\
\mathrm{CA} A^{\mathrm{Np}-1} \mathrm{~B} & \mathrm{CA}^{\mathrm{Np}-2} \mathrm{~B} & . & \mathrm{CA}^{\mathrm{Np}-\mathrm{Ne}_{B}}
\end{array}\right]}
\end{aligned}
$$

The state space portrayal of the framework when disturbances are included

$x(k+1)=A_{n} x(k)+B_{n 1} u(k)+B_{n 2} \theta(k)+B_{n 2} w(k)$

$$
y(k)=C_{n} \mathrm{x}(k)+D_{n 1} e(k)+D_{n 2} w(k)
$$

where $\mathrm{e}(\mathrm{k})=$ unmeasurable disturbances

$$
\mathrm{w}(\mathrm{k})=\text { measurable disturbances }
$$

With a specific end goal to settle on the best decision, a standard is relied upon to emulate the goal. The goal is related to an error function in light of the refinement between the coveted and the genuine reactions. This target work is frequently called the cost function $\mathrm{J}$, and the perfect control movement is found by constraining this cost function [15]. $\mathrm{J}=\sum_{\mathrm{i}=1}^{\mathbb{N}_{D}}\left(\left(y_{k+i}-\eta_{k+i}\right)^{T} \mathrm{Q}\left(y_{k+i}-\eta_{k+i}\right)+u_{k+i-1}{ }^{T} \mathrm{P}\right.$ $u_{k+i-1}+\Delta u_{k+i-1}^{T} R \Delta u_{k+i-1}$

Here $N_{p}$ is the prediction horizon $Q \in \mathbb{R}^{m \times m}, \mathrm{P}_{v} R \in \mathbb{R}^{r \times x}$ are symmetric and positive semi definite weighting matrices which are specified by the users.

\section{SIMULATION}

This section presented a simulation example to delineate the proposed model predictive control strategy for NCS in presence of time delay. Matlab based Truetime simulator is an effective tool which is used to implement real time networked system design. In this simulator, there are two types of Simulink blocks computer block and network block.In this simulation we assume that there is no packet droupout.

The discrete time model of servo system at a sampling time of $0.02 \mathrm{~s}$ is [1].

$$
G_{p}(z)=\frac{0.0540 \mathrm{z}^{-2}+0.115 \mathrm{z}^{-3}+0.001 \mathrm{z}^{-4}}{1-1.12 \mathrm{z}^{-1}-0.212 \mathrm{z}^{-2}+0.235 \mathrm{z}^{-3}}
$$

The state space representation of the servo system

$$
\mathrm{A}=\left[\begin{array}{cccc}
1.120 & 0.2130 & -0.3350 & 0 \\
1 & 0 & 0 & 0 \\
0 & 1 & 0 & 0 \\
0 & 0 & 1 & 0
\end{array}\right]
$$

$\mathrm{B}=\left[\begin{array}{c}0.5000 \\ 0 \\ 0 \\ 0\end{array}\right]$

$$
\mathrm{C}=\left[\begin{array}{llll}
0 & 0.1080 & 0.230 & 0.002
\end{array}\right] \quad \mathrm{D}=\left[\begin{array}{llll}
0 & 0 & 0 & 0
\end{array}\right]
$$

For the given framework, a simulated response was checked with a step input, and utilizing Simulink. The response found was as in figure 5 .

Figure 6 represents the network schedule of the system. A high values represent the sending or executing and a low values represent the idle condition of the network. The model predictive controller is applied to this servo system it is necessary to select an $(\mathrm{Np})$ prediction horizon and $(\mathrm{Nc})$ control horizon. The value of $\mathrm{Np}$ and $\mathrm{Nc}$ of the predictive controller is varying to improve the response of plant in the presence of delay.

All simulation results from figure 6 to figure 12 represent that time delay is varying from 0.010 0.085 and 0.065 . The system response degraded if time delay 


\section{Predictive Controller Design for Networked Control System}

increased. The set point of proposed model predictive controller is 10 .

\section{CONCLUSION}

In this research paper, a model predictive controller based networked control system has been designed and analyze the performance. The proposed predictive controller is useful for recovering the response of the servo system under the occurrence of delay. This strategy is beneficiary to find the projected control performance and system stability. In this design predictive controller is placed in place of the controller to predict the plant states at the current sampling time. The predictive controller parameters have been varied according to the system response. The advantage of the model predictive controller is verified by the simulation results.

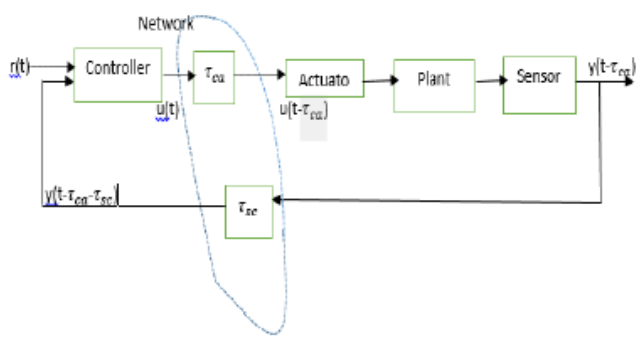

Figure 1 Networked closed loop system with communication delays Network

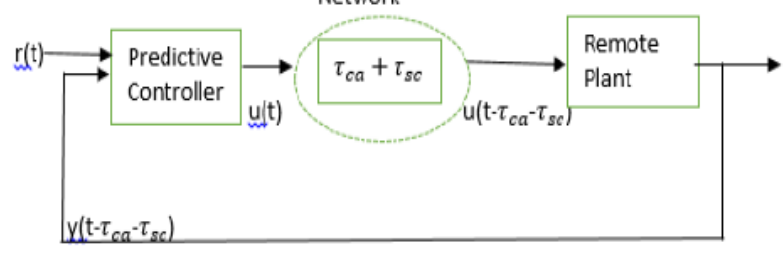

Figure 2 Networked control system with predictive controller

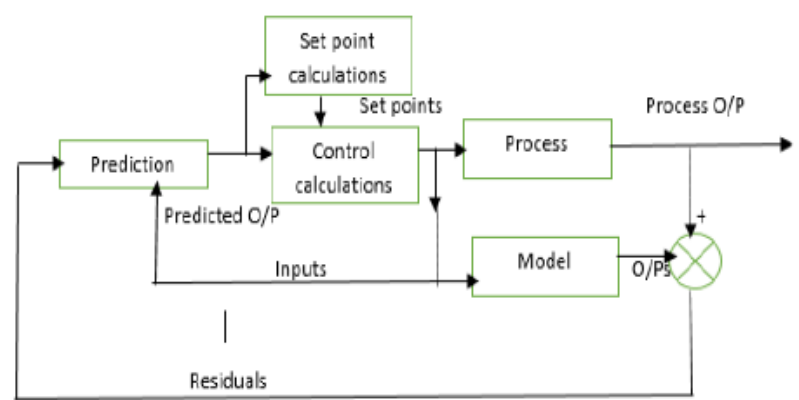

Figure 3 Diagram of a predictive controller

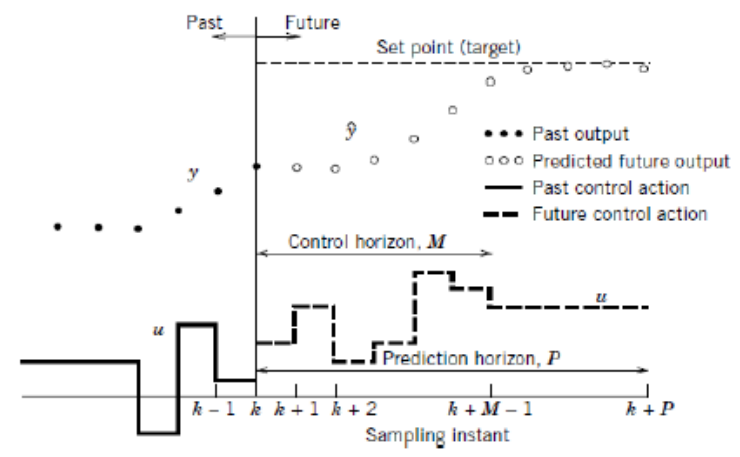

Figure 4 The basic concept for predictive control [2]

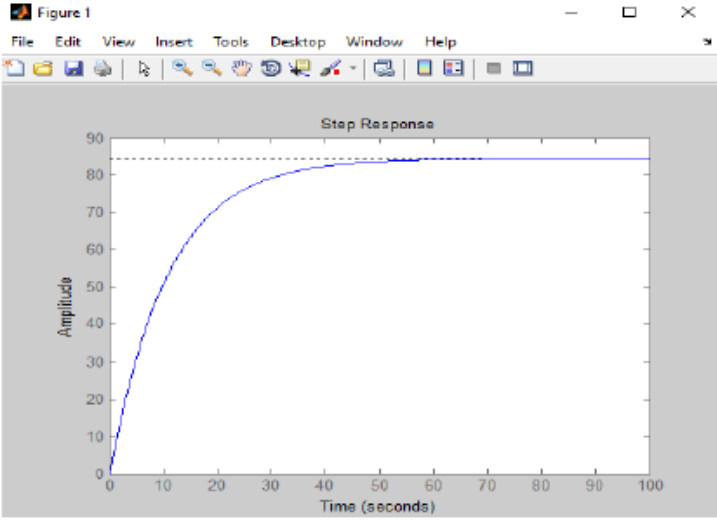

Figure 5. Step response of the servo system

Network Schedule

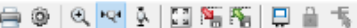

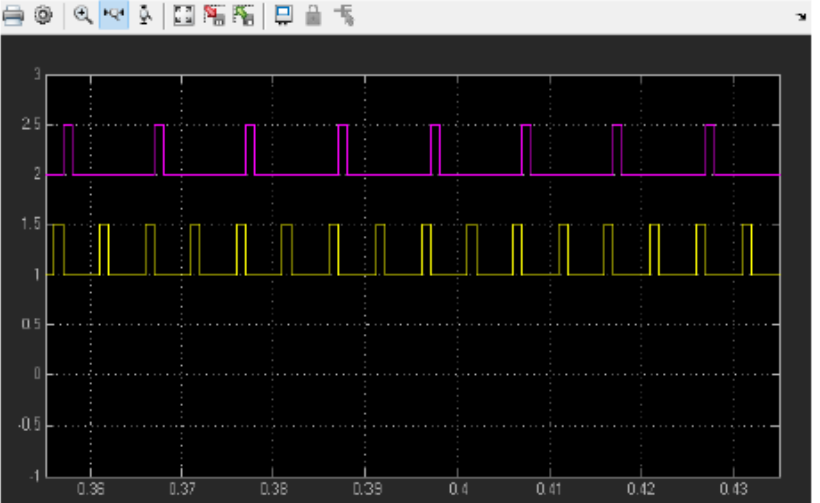

Figure 6. Network Schedule

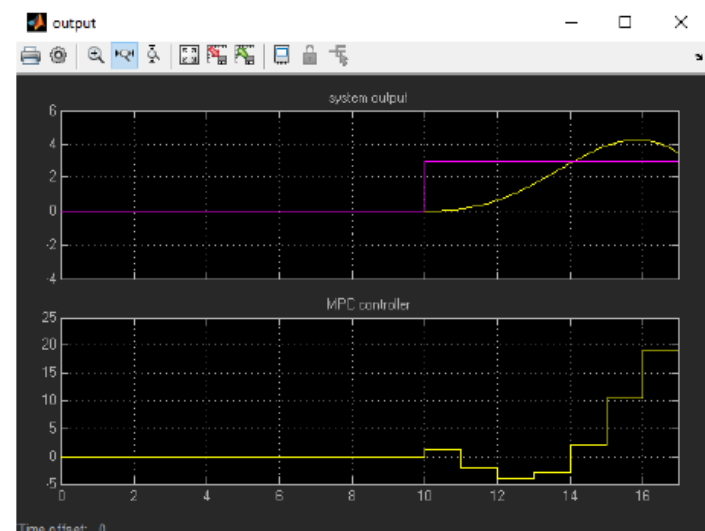

Figure 7. $\mathrm{Np}=5, \mathrm{Nc}=2$ Time delay $=0.065$, weight tuning $=0.8$

output

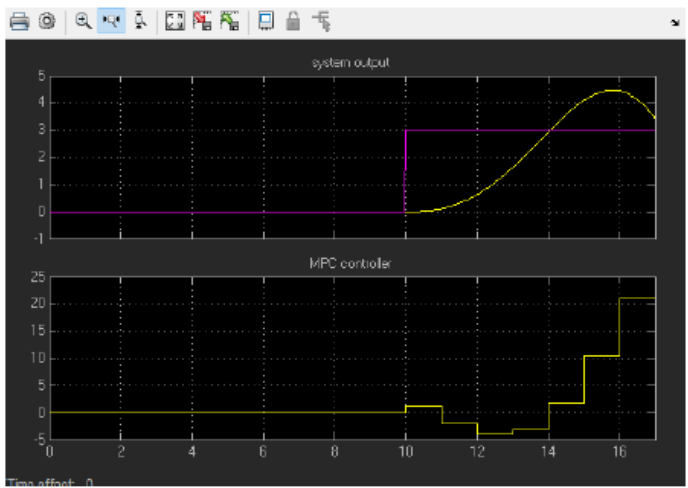

Figure $8 . \mathrm{Np}=5, \mathrm{Nc}=2$ Time delay $=0.085$ 
output
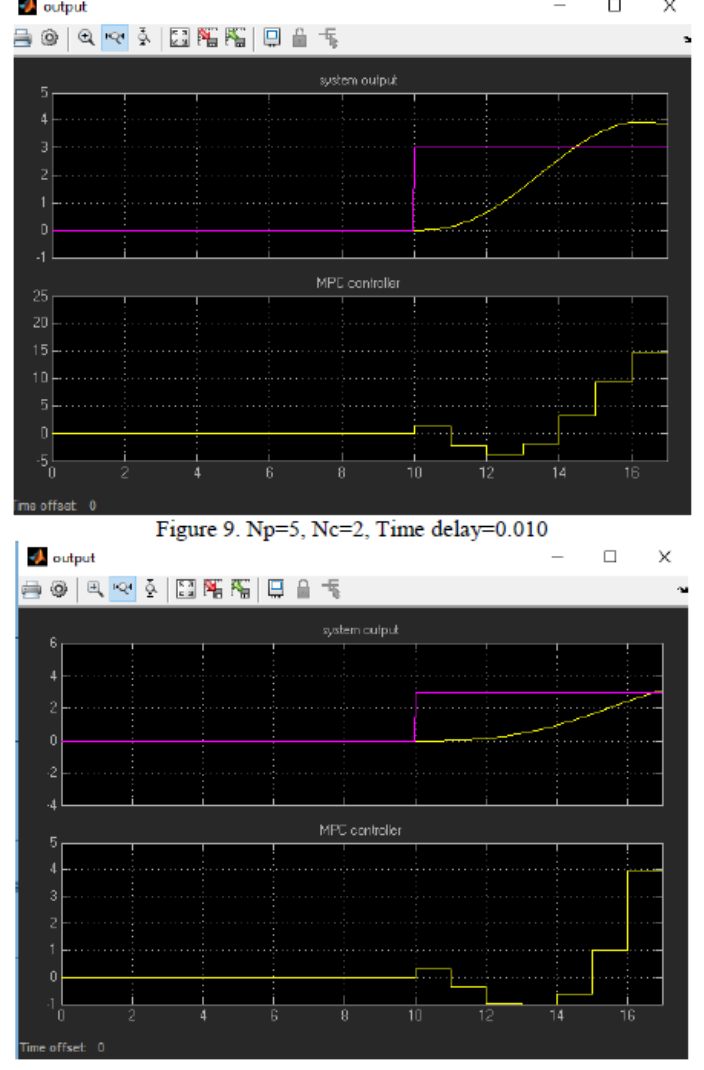

Figure 10. $\mathrm{Np}=7, \mathrm{Nc}=4$ Time delay $=0.080$, weight tuning $=0.6$

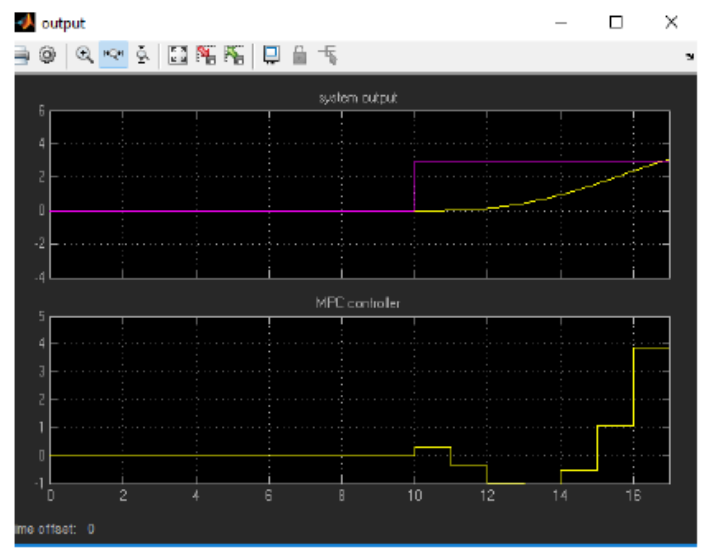

Doutput

Figure 11. $\mathrm{Np}=7, \mathrm{Nc}=4$, Time delay $=0.065$

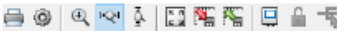

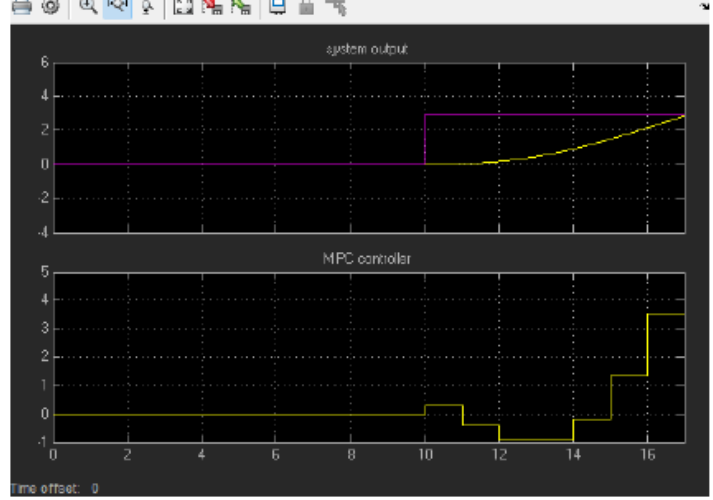

Figure 12. $\mathrm{Np}=7, \mathrm{Nc}=4$, Time delay $=0.010$

\section{REFERENCES}

1. Rongni Yang, Guo-Ping Liu, Clive Thomas, and Michael V. Basin, "Predictive Output Feedback Control for Networked Control Systems" ,IEEE Transactions on industrial electronics, vol.61, no.1, January 2014.

2. G. P. Liu, "Predictive Controller Design of Networked Systems with Communication Delays and Data Loss", IEEE Transactions on circuits and systems, vol.57, no.6, June 2010.

3. Hongbo Li, Zengqi Sun, and Mo-Yuen Chow, "State feedback controller design of networked control systems with time delay and packet dropout," Proceedings of the 17th World Congress the International Federation of Automatic Control Seoul, Korea, July 2008.

4. A. Onat, T. Naskali, E. Parlakay, and O. Mutluer, "Control over imperfect networks: Model-based predictive networked control systems,"mIEEE Trans. Ind. Electron., vol. 58, no. 3, pp. 905-913, Mar. 2011.

5. Han, C.-W., "Fuzzy Neural Network-based Time Delay Prediction for Networked Control Systems", Appl. Math Inf. Sci. 2014, 8, 407-413.

6. Tomas Estrada and Panos J. Antsaklis, "Performance of Model-Based Networked Control Systems with Discrete-Time Plants", 17th Mediterranean Conference on Control \& Automation Makedonia Palace, Thessaloniki, Greece, 2009.

7. L.A.Montestruque and P.J. Antsaklis, "Networked Control Systems: A model-based approach," 12th Mediterranean Conference on Controland Automation, June 2004.

8. Junfeng Zhang, Xianglei Jia, Ridong Zhang \& Yan Zuo, “A model predictive control framework for constrained uncertain positive systems",International Journal of system and science,vol.49,pp-884-896, 2017.

9. ZHOU Yuanqiang, LI Dewei, XI Yugeng, CEN Lihui, XU Yuli, GAN Zhongxue, "Distributed Model Predictive Control for Nonlinear Networked Systems with Asynchronous Communication", Proceedings of the 36th Chinese Control Conference 2017.

10. J. Wu, L. Zhang, T. Chen, "Model predictive control for networked control systems", International Journal of Robust and Nonlinear Control,vol. 19, no. 9, 2009, pp: 1016-1035.

11. Z. Wang, W. Pan, and G. Guo, "Stability of Model-Based Networked Control System with Quantized Feedback," in Proceedings of the 3rdInternational Conference on Innovative Computing Information and Control, Dalian, Liaoning, 2008.

12. Yang, R.-N.; Liu, G.-P.; Shi, P.; Thomas, C.; Basin, "Predictive output feedback control for networked control systems", IEEE Trans. Ind.Electron. 2014, 61, 512-520

13. Song, H.-B.; Liu, G.-P.; Yu, L., "Networked predictive control of uncertain systems with multiple feedback channels", IEEE Trans. Ind.Electron. 2013, 60, 5228-5238.

14. C.F.Carunyu,C.Lazar, "Robustly stabilising model predictive control design for networked control systems with an application to direct current motors" IET control theory and applications 2012 vol.6, pp:943-952.

15. David-Henriet, X., Hardouin, L., Raisch, J., \& Cottenceau, B. , "Model predictive control for discrete event systems with partial synchronization", Automatica, 70, 9-13 2016.

16. A. Cervin, D. Henriksson, M. Ohlin, TrueTime 2.0 beta $5-$ Reference Manual, Department of Automatic Control, Lund University, Sweden, June 2010 